Surgery is disadvantaged when it comes to health research. Unlike trials of new drug treatments, research on surgical procedures has no natural sponsor. The lack of strict regulatory mechanisms for the approval of surgical procedures and devices in most countries leads to a situation where large randomised trials are not necessary for surgical interventions to be adopted. How surgical procedures and perioperative care are provided by different surgeons or hospitals varies enormously, leading to an "expertise bias" that may make a valid randomised controlled trial impossible in many circumstances ${ }^{5}$ and in others may limit the generalisability of results to other care settings.

Problems with evidence based surgery notwithstanding, we would argue that the immediate challenge to improving the quality of surgical care is not discovering new knowledge, but rather how to integrate what we already know into practice. Lassen et $\mathrm{al}^{1}$ and others remind us that much may be achieved simply by raising the quality of surgical care according to existing evidence. In many ways, this is a far more difficult task than simply doing more research. Translating best evidence into surgical practice will require the engagement of large numbers of individual practitioners, in a multitude of healthcare contexts, and using a variety of techniques.

Evidence from before and after studies in several countries shows that surgeons' behaviour can indeed be changed and the quality of surgical care can be improved. In Norway, surgeons concerned with poor outcomes of surgery for rectal cancer initiated a multifaceted intervention in 1994 to improve the quality of care according to best practices. This initiative led to a voluntary registry of treatment for rectal cancer with feedback to hospitals as well as postgraduate courses for surgeons and pathologists on optimal surgical techniques and on interpreting pathological tests, reorganisation at specific regional hospitals and specialist gastrointestinal surgeons of surgery for rectal cancer, and access to an opinion leader for surgeons both locally and regionally. ${ }^{6}$ After the intervention, the rate of local recurrence of rectal cancer fell from 28\% to $8 \%$, and five year survival increased from $55 \%$ to $71 \%{ }^{7}$

In the United States, the Northern New England Cardiovascular Disease Study Group initiated a regional intervention in 1990 to improve the outcomes of coronary artery bypass graft surgery. This intervention comprised anonymous feedback to surgeons of risk adjusted outcome data, training in continuous quality improvement, and a series of site visits by surgeons to observe processes of care in other settings. After the intervention, mortality from coronary artery bypass graft surgery decreased by $24 \%{ }^{8}$

A key factor underlying successful programmes for quality improvement in surgery seems to be the engagement of individual surgeons both locally and regionally, through developing communities of practice. ${ }^{9}$ We now know more about what it takes to translate evidence into knowledge for practising surgeons. Innovative interventions that bring surgeons together may require substantial investment, but they will be worth while if they deliver evidence based surgery.

David R Urbach assistant professor of surgery and health policy, management and evaluation

University of Toronto and Cancer Care Ontario, 200 Elizabeth Street, 10 NU-214, Toronto, ON, Canada M5G 2C4

Nancy N Baxter assistant professor of surgery

Division of Surgical Colon and Rectal Surgery, Comprehensive Cancer Center, University of Minnesota, Minneapolis, MN 55455 , USA

(baxte025@umn.edu)

Competing interests: None declared.

1 Lassen K, Hannemann P, Ljungqvist O, Fearon K, Dejong CHC, von Meyenfeldt MF, et al. Patterns in current perioperative practice: survey of Meyenfeldt MF, et al. Patterns in current perioperative practice: survey of
colorectal surgeons in five northern European countries. BMJ 2005;330:1420-1.

2 Horton R. Surgical research or comic opera: questions, but few answers. Lancet 1996;347:984-5.

3 Solomon MJ, McLeod RS. Should we be performing more randomized controlled trials evaluating surgical operations? Surgery 1995; 118:459-67.

4 Fingerhut A, Borie F, Dziri C. How to teach evidence-based surgery. World J Surg 2005. Published online 21 April 2005. www.springerlink.com/ media/788Y661VQJ3KQVT79TRN/Contributions/N/5/2/3/ N523841J63551747 html/fulltext.html (accessed 10 June).

5 Devereaux PJ, Bhandari M, Clarke M, Montori VM, Cook DJ, Yusuf S, et al. Need for expertise based randomised controlled trials. BMJ 2005; et al. Need

6 Wibe A, Møller B, Norstein J, Carlsen E, Wiig JN, Heald RJ, et al. A national strategic change in treatment policy for rectal cancerimplementation of total mesorectal excision as routine treatment in Norway. A national audit. Dis Colon Rectum 2002;45:857-66.

7 Wibe A, Eriksen MT, Syse A, Myrvold HE, Søreide O. Total mesorectal excision for rectal cancer-what can be achieved by a national audit? Colorectal Dis 2003;5:471-7.

8 O'Connor GT, Plume SK, Olmstead EM, Morton JR, Maloney CT, Nugent WC, et al. A regional intervention to improve the hospital mortality associated with coronary artery bypass graft surgery. The Northern New England Cardiovascular Disease Study Group. JAMA 1996;275:841-6.

9 Gagliardi A, Ashbury FD, George R, Irish J, Stern HS, Cancer Care Gagliardi A, Ashbury FD, George R, Irish J, Stern HS, Cancer Care
Ontario. Improving cancer surgery in Ontario: recommendations from a strategic planning retreat. Can J Surg 2004;47:270-6.

\title{
Buruli ulcer
}

\section{A neglected but treatable disease that unnecessarily scars African children for life}

$\mathrm{B}$ uruli ulcer, caused by infection with Mycobacterium ulcerans, is unfamiliar because it is common only among children in rural tropical areas, mainly in west Africa. Ghana, Côte d'Ivoire, and Benin are among the worst affected. ${ }^{1}$ It is named Buruli after a county in Uganda. It is an easily treatable disease as common as tuberculosis in areas where it is endemic. ${ }^{\mathrm{w1}}$ In endemic areas in Ghana point prevalence has been estimated at 150-180/100 000 population, and in southern Benin, a detection rate of 20/100 000 per year has been reported."2 w3 Lack of treatment leaves children with large disfiguring scars and occasionally with joint deformities, loss of sight, loss of breast or scrotal tissue, and even amputations.

Ulcers (figure) can reach a dramatic size, particularly in the oedematous form, which spreads rapidly over large parts of the limbs, trunk, or head. How infection is transmitted remains a mystery; it may be through infection of simple grazes on the skin by water

\section{Additional references w1-w6 are on bmj.com}




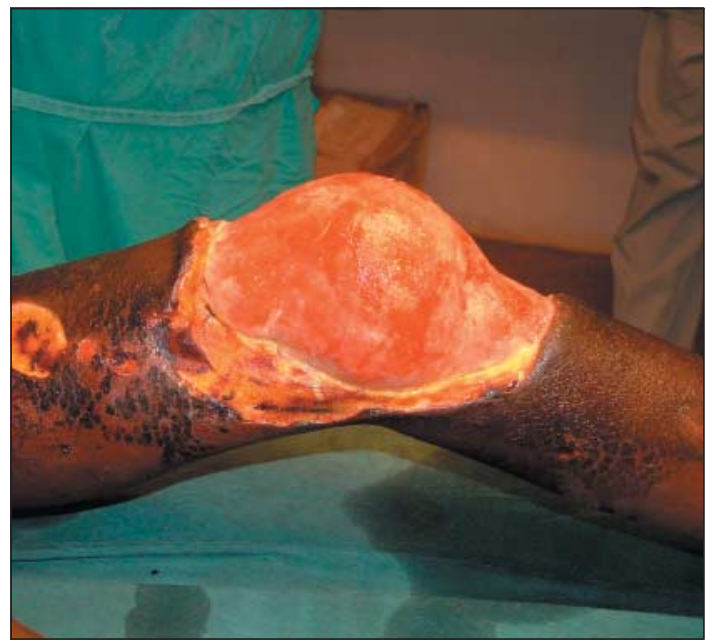

Buruli ulcer on the knee of an 8 year old Ghanaian boy

from rivers, ponds, or even mud, the rainy season being the time when most infections occur. Water insects (Naucoridae and Belostomatidae) have been incriminated, but their bites have not yet been shown to transmit infection to humans. Water insects from rivers in Côte d'Ivoire can bite and infect mouse tails in the laboratory, but $M$ ulcerans has not been cultured directly from the environment. ${ }^{2}$ Ongoing studies of differences in environmental water between endemic and non-endemic areas may shed more light on the highly focal epidemiology of the disease.

$M$ ulcerans replicates in subcutaneous tissue where the temperature approaches the optimum of $30-33^{\circ} \mathrm{C}$ for in vitro culture. It was first cultured in Melbourne in 1947 when an incubator broke down and the temperature dropped to this level, leading eventually to the establishment of a link between the organism and the disease known there as Bairnsdale ulcer. ${ }^{\mathrm{w} 4}$

Why temperate southeastern Australia should be the exception in its epidemiology is a mystery, but researchers in that country have taken a lead in investigating the problem. They have recently collaborated in sequencing the genome of $M$ ulcerans, ${ }^{3}$ which takes us to the heart of what makes it unique among mycobacteria. It causes disease through production of a potent tissue destructive toxin called mycolactone. ${ }^{4}$ This toxic macrolide prevents phagocytosis of live organisms and may delay the development of an inflammatory response as well as causing cell death by apoptosis. ${ }^{5}$

Infected subcutaneous tissue shows extensive fatty necrosis with numerous clumps of extracellular mycobacteria but no inflammatory reaction in the immediate vicinity. ${ }^{\mathrm{w} 5}$ Detailed study of the genome has found that mycolactone is encoded by genes for three polyketide synthases and other enzymes on two identical copies of a plasmid separate from the main DNA sequence. ${ }^{6}$ Further work will possibly generate ways of inhibiting the toxin itself or pathways involved in its production. Knowledge of the genome should increase understanding of the molecular biology of the organism, leading perhaps to revelation of the mode of transmission of infection and the possibility of developing vaccines.

Traditionally treatment has relied on complete excision of infected tissue, but we now know that normal looking skin around a lesion contains organisms. As access to surgery in rural tropical areas is problematic the focus is shifting to treatment with rifampicin and streptomycin combined with much smaller surgical intervention. Years of in vitro and animal model studies have established this combination of antibiotics as the most effective way of killing $M$ ulcerans, and more recent work shows that the organisms can be killed in human tissue by treatment for at least four weeks. ${ }^{78} \mathrm{~A}$ prospective but uncontrolled study of patients in Benin according to guidelines from the World Health Organization has shown that most are cured by treatment with rifampicin and streptomycin for eight weeks with less than $3 \%$ recurrences. ${ }^{9}$

If inhibition of mycolactone production alone is sufficient to allow the immune response to control infection in humans is not known, but evidence shows that nearly one third of early lesions heal without any treatment. ${ }^{10}$ A superficial infection, Buruli ulcer may be susceptible to topical treatment alone. Creams generating topical nitrogen oxide promoted healing in one small study, and nitric oxide killed $M$ ulcerans in vitro. ${ }^{112}$ Phenytoin powder probably does not kill the organism, but it seemed to promote healing, perhaps through acceleration of fibrogenesis. ${ }^{\mathrm{w} 6}$

Future research needs to concentrate on three main areas-the mode of transmission of the disease; the effectiveness of antibiotics and the best way of using them; and characterisation of proteins specific to $M$ ulcerans through knowledge of the genome sequence in order to develop an effective vaccine.

Mark Wansbrough-Jones consultant physician Richard Phillips Wellcome Trust research fellow

St George's Hospital Medical School, London SW17 0RE (wansbrou@sghms.ac.uk)

Competing interests: None declared.

1 Asiedu K, Scherpbier R, Raviglione M. Buruli ulcer:Mycobacterium ulceran s infection. Geneva: World Health Organization, 2000. www.who.int/gtb infection. Geneva: World Health Organization, 2000. www.who.int/gtbAuruli/pubs 2005).

2 Marsollier L, Aubry J, Saint-Andre JP, Robert R, Legras P, Manceau AL, et al. [Ecology and transmission of Mycobacterium ulcerans]. Pathol Biol (Paris) 2003;51:490-5.

3 Stinear TP. The complete genome sequence of Mycobacterium ulcerans strain Agy99. Report of the 8th annual WHO advisory group meeting on Buruli ulcer. 2005. (In press.)

4 George KM, Chatterjee D, Gunawardana G, Welty D, Hayman J, Lee R, et al. Mycolactone: a polyketide toxin from Mycobacterium ulcerans required for virulence. Science 1999;283:854-7.

5 George KM, Pascopella L, Welty DM, Small PL. A Mycobacterium ulcerGeorge KM, Pascopella L, Welty DM, Small PL. A Mycobacterium ulcer-
ans toxin, mycolactone, causes apoptosis in guinea pig ulcers and tissue ans toxin, mycolactone, causes apoptosis in
culture cells. Infect Immun 2000;68:877-83.

6 Stinear TP, Mve-Obiang A, Small PL, Frigui W, Pryor MJ, Brosch R, et al. Giant plasmid-encoded polyketide synthases produce the macrolide toxin of Mycobacterium ulcerans. Proc Natl Acad Sci USA 2004;101:1345-9.

7 Dega H, Bentoucha A, Robert J, Jarlier V, Grosset J. Bactericidal activity of rifampin-amikacin against Mycobacterium ulcerans in mice. Antimicrob Agents Chemother 2002;46:3193-6.

8 Etuaful S, Carbonnelle B, Grosset J, Lucas S, Horsefield C, Phillips R, et al. Bactericidal activity of rifampicin and streptomycin treatment for early human
M. ulcerans lesions. Report of the 7th Annual WHO Advisory Group M. ulcerans lesions. Report of the 7th Annual WHO Advisory Group
Meeting on Buruli ulcer. 2004. p110. www.who.int/gtb-buruli/ Meeting on Buruli ulcer. 2004. p110. Www.who.int/gtb-buruli/
publications/PDF/WHO-CDS-CPE-GBUI-2004.9.pdf (accessed $21 \mathrm{Apr}$
2005). 2005)

9 Chauty A. Drug treatment in Benin. Report of the 8th Annual WHO Advisory Group Meeting on Buruli ulcer. 2005. (In press.)

10 Revill WD, Morrow RH, Pike MC, Ateng J. A controlled trial of the treatment of Mycobacterium ulcerans infection with clofazimine. Lancet $1973 ; 2: 873-7$

11 Phillips R, Adjei O, Lucas S, Benjamin N, Wansbrough-Jones M. Pilot randomized double-blind trial of treatment of Mycobacterium ulcerans disease (Buruli ulcer) with topical nitrogen oxides. Antimicrob Agents Chemother 2004:48:2866-70.

12 Phillips R, Kuijper S, Benjamin N, Wansbrough-Jones M, Wilks M, Kolk AH. In vitro killing of Mycobacterium ulcerans by acidified nitrite. Antimicrob Agents Chemother 2004;48:3130-2. 